\title{
Análise da antibioticoprofilaxia em procedimentos cirúrgicos eletivos
}

\author{
Dryelle Cristina da Costa Feitoza ${ }^{1}$ \\ GleyceMikaelle Costa Quirino ${ }^{2}$ \\ Sônia Maria Alves Gomes ${ }^{3}$ \\ Ruth Silva Matos ${ }^{4}$ \\ Alexandra Isabel de Amorim Lino ${ }^{5}$ \\ Raniele Barros de Oliveira ${ }^{6}$ \\ Lauane Rocha Itacarambi ${ }^{7}$ \\ Jacqueline Ramos de Andrade Antunes Gomes ${ }^{8}$ \\ Samir Miranda Bitencourt ${ }^{9}$ \\ Suely Moreira Do Nascimento Araujo ${ }^{10}$
}

\footnotetext{
${ }^{1}$ Residente do programa de residência uniprofissional de enfermagem em centro cirúrgico ESCS/FEPECS/SESDF

${ }^{2}$ Residente do programa de residência uniprofissional de enfermagem em centro cirúrgico ESCS/FEPECS/SESDF

${ }^{3}$ Enfermeira Especialista em Auditoria em Saúde pela UFG, especialista em Centro Cirúrgico pela Faculdade de Enf. LuisaMarillac

${ }^{4}$ Residente do programa de residência uniprofissional de enfermagem em centro cirúrgico ESCS/FEPECS/SESDF

${ }^{5}$ Enfermeira Mestre em enfermagem pela Universidade de Brasília,Enfermeira da Secretaria de Estado de Saúde do Governo do Distrito Federal

${ }^{6}$ Residente do programa de residência uniprofissional de enfermagem em centro cirúrgico ESCS/FEPECS/SESDF.

${ }^{7}$ Residente do programa de residência uniprofissional de enfermagem em centro cirúrgico ESCS/FEPECS/SESDF.

${ }^{8}$ Enfermeira coordenadora do Programa de Residência de Enfermagem em Centro Cirúrgico ESCS/FEPECS/SES/DF.

9 Enfermeiro Preceptor do Programa de Residência de Enfermagem em Centro Cirúrgico ESCS/FEPECS/SES/DF.

${ }^{10}$ Enfermeira Preceptora do Programa de Residência de Enfermagem em Centro Cirúrgico ESCS/FEPECS/SES/DF.
}

\section{RESUMO}

Introdução: A antibioticoprofilaxia em ambiente cirúrgico visa reduzir as infecções de sítio cirúrgico (ISC), sendo um fator adjuvante na prevenção. Objetivou-se analisar o uso de antibioticoprofilaxia em procedimentos cirúrgicos eletivos em um hospital público do Distrito Federal (DF).Métodos: Trata-se de uma pesquisa transversal, retrospectiva com abordagem quantitativa realizada em prontuários de procedimentos cirúrgicos eletivos em um hospital público do DF. O tamanho amostral foi estimado por meio de fórmula através de 320 prontuários. Resultados: Prevaleceram procedimentos cirúrgicos realizados pela cirurgia 
geral $43,8 \%$. Quanto ao potencial de contaminação da incisão cirúrgica a prevalência de $53,8 \%$ cirurgias potencialmente contaminadas. Houve a utilização de antibioticoprofilático em $98,4 \%$ dos procedimentos, sendo a cefazolina 50,6\% antibiótico de escolha. A média de internação foi 2,91 dias com desvio de 3,65. Verificou-se que 1,6\% dos participantes apresentaram ISC. Conclusão: conclui-se que 1,6\% da amostra apresentaram ISC, assim a utilização de antibioticoprofilaxia foi considerada adequada por conta do percentual de procedimentos que a utilizaram $98,4 \%$ que se enquadravam em procedimentos potencialmente contaminados, sendo indispensável o uso de antimicrobiano.

Palavras Chaves: Antibioticoprofilaxia; Infecção da Ferida Cirúrgica; Enfermagem Perioperatória.

\title{
Analysis of antibiticoprophylaxis in elective surgical procedures
}

\begin{abstract}
Introduction: Antibiotic prophylaxis in the surgical environment aims to reduce surgical site infections (SSI), being an adjuvant factor in prevention. The objective of this study was to analyze the use of antibiotic prophylaxis in elective surgical procedures in a public hospital in the Federal District (DF).Methods: This is a cross-sectional, retrospective study with a quantitative approach carried out on medical records of elective surgical procedures in a public hospital in the DF. The sample size was estimated using a formula using 320 medical records. Results: Surgical procedures performed by general surgery prevailed $43.8 \%$. As for the potential for contamination of the surgical incision, the prevalence of $53.8 \%$ potentially contaminated surgeries. Prophylactic antibiotics were used in $98.4 \%$ of the procedures, with cefazolin being $50.6 \%$ of choice. The average hospital stay was 2.91 days with a deviation of 3.65. It was found that $1.6 \%$ of the participants had SSI. Conclusion: it is concluded that $1.6 \%$ of the sample had SSI, so the use of antibiotic prophylaxis was considered adequate due to the percentage of procedures that used it, $98.4 \%$ that fit into potentially contaminated procedures, being essential the use of antimicrobials .
\end{abstract}

KeyWords: Antibiotic prophylaxis; Surgical Wound Infection; Perioperative Nursing.

\section{INTRODUÇÃO}

Dentro da perspectiva de garantia da segurança do paciente, através de ações que visem reduzir os riscos de eventos adversos em ambiente hospitalar, a Organização Mundial da Saúde (OMS) estabeleceu desafios globais, dos quais a redução da infecção por meio da promoção de cirurgia segura, mediante ações pré, durante e após o ato cirúrgico constituiu fator de destaque ${ }^{1}$.

Dessa maneira em meio a necessidade cada vez maior de promover melhorias na qualidade e segurança das intervenções cirúrgicas, esse desafio objetivou aumentar os padrões 
de qualidade desejados em serviços de saúde, contemplando dentre outros a prevenção de infecções de sítio cirúrgico ${ }^{1}$.

As infecções de sítio cirúrgico (ISC) hospitalares, apresentam uma incidência aproximada de 2 a $11 \%$ em todas as ações cirúrgicas. Para mais, além do ônus com tratamento e permanência prolongada, a persistência dessas infecções aumenta também a chance de mortalidade $\mathrm{e}^{2,3,4}$

Em se tratando de cirurgias eletivas, essas são compreendidas como procedimentos cirúrgicos de média e alta complexidade de todas as especialidades médicas, agendadas, sem caráter de urgência e emergência ${ }^{5}$.

É no período intraoperatório que acontece a maioria das ISC, assim é necessário um olhar mais atento quanto aos cuidados profiláticos em cirurgias eletivas, já que, apresentam maior eficácia comparados com cirurgias de urgência e emergência, e seria inaceitável sua ausência ou uso inadequado. Assim, o uso de antibióticos em cirurgias eletivas contribui para a diminuição da bacteremia e, consequentemente, o número de microrganismos próximos ao local da incisão ${ }^{6,7}$.

No controle e prevenção de infecção, além de outros fatores, o uso de antibióticos constitui elemento fundamental, uma vez que, atua inibindo diferentes tipos de microrganismos patogênicos, sendo esses classificados em antibacterianos, antifúngicos, antiparasitários e antivirais ${ }^{8}$.

Diante da atuação no controle de microrganismos, a profilaxia antimicrobiana em ambiente cirúrgico visa reduzir a incidência de ISC, sendo considerada como um fator adjuvante na prevenção das infecções ${ }^{9}$.

Nesse caso, a adoção de algumas recomendações é necessária para uma profilaxia adequada, como: ter indicação apropriada; determinar a provável microbiota numa infecção pós-operatória; escolher o antimicrobiano menos tóxico e o de menor custo entre os de igual 
eficácia e avaliar o risco de toxicidade, desenvolvimento de resistência e custo do antibiótico antes da indicação da profilaxia antimicrobiana ${ }^{9}$.

Com o intuito de assegurar o controle de tal infecção, atingir resultados clínicos ideais em cirurgias, reduzir eventos adversos a medicamentos, atenuar os custos relacionados à infecção do sítio cirúrgico, minimizar a patogênese de cepas resistentes a antimicrobianos, a aderência a antibioticoprofilaxia eficaz e administração no tempo correto, todos esses cuidados constituem mecanismos que asseguram o cuidado fundamental ${ }^{10,11}$.

A enfermagem e a equipe médica possuem papel primordial no surgimento da ISC, atuando na prevenção de fatores de risco modificáveis através da adoção de medidas, como a limpeza e desinfecção de equipamentos e do ambiente, higienização das mãos, diminuição do tempo pré-operatório, banho pré-operatório, diminuição da colonização bacteriana da pele, realização da tricotomia e controle de temperatura ${ }^{12,13}$.

Dessa forma, este estudo tem por objetivo analisar a profilaxia antimicrobiana em cirurgias eletivas e seus resultados para identificação de possíveis erros preveníveis pelo ambiente da pesquisa e demais que os apresentarem.

\section{MÉTODO}

Trata-se de pesquisa transversal, retrospectiva com abordagem quantitativa. A pesquisa foi conduzida no período de julho a dezembro de 2020 , realizada em um hospital público do Distrito Federal (DF). A população constituiu-se de 1.896 prontuários de pacientes de ambos os sexos, submetidos a procedimentos cirúrgicos eletivos do período de maio de 2019 a maio de 2020.

O tamanho amostral foi estimado por meio da fórmula para populações finitas para estudos transversais. Foram considerados como parâmetros o coeficiente de confiança de $95 \%$ $(1,96)$, erro tipo 1 de $5 \%$, o erro amostral de 5\%, proporção de ocorrência do fenômeno, como 
regra geral utilizou-se, $\mathrm{p}=50 \%$, pois não tinha informações sobre o valor esperado. Assim, a partir da aplicação da fórmula a amostra estimada foi em 320 prontuários, no qual foram incluídos prontuários de pacientes acima de 18 anos e excluídos prontuários de pacientes submetidos a urgência ou emergência, em uso de antibiótico no pré-operatório mediato e prontuários com ausência de informação.

As informações colhidas para o preenchimento dos formulários foram obtidas através de informações contidas nos prontuários eletrônicos dos pacientes e check-list de cirurgia segura. Foi utilizado um formulário estruturado e preenchido pela pesquisadora responsável, as variáveis do estudo foram divididas em: clínica do procedimento cirúrgico, classificação da cirurgia por potencial de contaminação, administração de antibiótico e qual foi utilizado, tempo de internação e presença ou não de infecção de sitio cirúrgico.

Os dados foram tabulados e analisados no Statistical Package for the Social Sciences (SPSS), versão 20.0. Foi realizada estatística analítica descritiva: frequência simples, desvio padrão, média, valores máximos e valores mínimos.

Foram elaboradas tabelas, a fim de apresentar os resultados com detalhamento para melhor compreensão. Os resultados foram analisados e discutidos com base na literatura científica pertinente sobre a temática.

Este estudo respeitou as exigências da Resolução $n^{\circ} 466 / 2012$ CNS/MS ${ }^{14}$ e foi aprovado através do parecer 4.217.878.

\section{RESULTADOS E DISCUSSÕES}

O estudo compreendeu um total de 320 prontuários de pacientes para análise. Das variáveis analisadas na tabela 1 segundo a clínica dos procedimentos realizados, houve predomínio de $140(43,8 \%)$ dos procedimentos realizados pela Cirurgia geral, seguindo 73 
$(22,8 \%)$ dos procedimentos da cirurgia ginecológica e obstétrica, 17 (5,3\%) mastologia, 45 $(14,1 \%)$ ortopédica e $45(14,1 \%)$ cirurgia urológica.

Em concordância ao estudo de Gouvea ${ }^{15}$, a especialidade de cirurgia geral $(53,1 \%)$ e cirurgia ginecológica $(18,8 \%)$ obtiveram percentual considerável sendo as clinicas com maior número de procedimentos realizados.

Tabela 1 - Caracterização dos participantes da pesquisa segundo a clínica dos procedimentos realizados. Brasília-DF-2020. $(\mathrm{n}=320)$.

\begin{tabular}{lcc}
\hline Variáveis & F & \% \\
\hline Geral & 140 & 43,8 \\
Ginecológica e obstétrica & 73 & 22,8 \\
Mastologia & 17 & 5,3 \\
Ortopedia & 45 & 14,1 \\
Urologia & 45 & 14,1 \\
\hline
\end{tabular}

Fonte: dados da pesquisa.

Quanto a classificação do potencial de contaminação, percebe-se na tabela 2, a prevalência de $172(53,8 \%)$ cirurgias potencialmente contaminadas, enquanto que $88(27,5 \%)$ representam cirurgias limpas, seguidos por cirurgias contaminadas $54(16,9 \%)$ e cirurgias infectadas $6(1,9 \%)$.

Para Tostes ${ }^{16}$, também teve achados semelhantes com prevalência de cirurgias potencialmente contaminadas e cirurgias limpas. Sabe-se que as ISC devem ser analisadas conforme o potencial de contaminação da ferida cirúrgica, entendido como o número de microrganismos presentes no tecido a ser operado. Então o risco de infecção é tanto maior quanto maior é o potencial de contaminação, razão pela qual se torna imperativo o uso de antimicrobianos. 
Segundo Comhupes ${ }^{17}$, indica-se antibioticoprofilaxia cirúrgica quando a chance de infecção incisional for $>5 \%$, ou seja, basicamente nas cirurgias contaminadas ou potencialmente contaminadas.

Nas cirurgias limpas, a profilaxia antibiótica está indicada nas situações onde a infecção seria clinicamente desastrosa, na cirurgia de urgência ou quando o paciente tem algum dos seguintes fatores de risco: cirurgia gastroduodenal em uso de droga que diminui o pH gástrico, desnutrição, idade >70 anos, imunodepressão, valvopatia reumática, diabetes descompensada, obesidade mórbida, comorbidades, icterícia obstrutiva ou coledocolitíase/colangite prévias ${ }^{17}$.

Tabela 2- Caracterização das cirurgias por potencial de contaminação da incisão cirúrgica. Brasília-DF- 2020. $(\mathrm{n}=320)$.

\begin{tabular}{llc}
\hline Variáveis & F & \% \\
\hline Limpa & 88 & 27,5 \\
Potencialmente contaminada & 172 & 53,8 \\
Contaminada & 54 & 16,9 \\
Infectada & 6 & 1,9 \\
\hline
\end{tabular}

Fonte: dados da pesquisa

A Tabela 3 informa sobre a utilização de antibiótico no intraoperatório, com representatividade de $315(98,4 \%)$ da amostra. Foi observado por outros autores, que a utilização de antibioticoprofilático esteve presente em 91,8\% dos casos, com taxas variando de $81,4 \%$ a $97,5 \%{ }^{16}$.

Como a profilaxia antimicrobiana é uma das medidas indispensáveis na prevenção das ISC, a não administração nos casos que possuem indicação é inaceitável e pode contribuir 
para o aumento da incidência das infecções relacionadas à assistência à saúde e comprometer a qualidade da assistência e a segurança do paciente ${ }^{18}$.

Tabela 3 - Identificação dos participantes da pesquisa segundo a utilização de antibiótico profilaxia. Brasília-DF - 2020. $(n=320)$.

\begin{tabular}{lcc}
\hline Variáveis & F & $\%$ \\
\hline Antibioticoprofilaxia & & \\
Sim & 315 & 98,4 \\
Não & 5 & 1,6 \\
\hline
\end{tabular}

Fonte: dados da pesquisa.

Em relação aos antibióticos profiláticos utilizados, a tabela 4 informa que 162(50,6\%) pacientes receberam cefazolina, $110(34,4 \%)$ clindamicina e $12(3,8 \%)$ ciprofloxacino.

Evidências apontam para a utilização das cefalosporinas em 93,60\% e 90,15\% nos procedimentos cirúrgicos, respectivamente ${ }^{16,18}$.

As cefalosporinas de primeira e segunda geração são realmente as mais difundidas, sendo a Cefazolina amplamente utilizada e geralmente considerada como o agente de primeira escolha para profilaxia. É eficaz contra muitos microrganismos Gram-positivos e Gramnegativos e também apresenta características de segurança como farmacocinética e um custo razoável por dose ${ }^{19}$.

Em relação ao predomínio do uso das cefalosporinas, no contexto internacional e nacional, a prática de seleção do antimicrobiano também segue essa tendência de uso, sendo os agentes profiláticos cirúrgicos amplamente utilizados e recomendados ${ }^{16}$.

Tabela 4 - Caracterização dos participantes da pesquisa segundo o antibiótico profilático utilizado. Brasília-DF - 2020. (n=317).

\begin{tabular}{lcc}
\hline Variáveis & F & $\%$ \\
\hline Cefazolina & 162 & 50,6 \\
Clindamicina & 110 & 34,4
\end{tabular}


Ciprofloxacino

Ampicilina+Sulbactam

Metronidazol + ciprofloxacino

Metronidazol + cefitraxona

Metronidazol + ciprofloxacino + cefazolina

Metronidazol + ceftriaxona + cefazolina
6

6

1,9

3

0,9

6

\section{Fonte: dados da pesquisa}

Houve associação entre antibióticos profiláticos como ampicilina e sulbactam em 12 $(3,8 \%)$ pacientes, o uso das associações (metronidazol + ciprofloxacino), (metronidazol e ceftriaxona), (metronidazol + ceftriaxona + cefazolina) em $6(1,9 \%)$ pacientes cada e metronidazol associado a ciprofloxacino e cefazolina em $3(0,9 \%)$.

Segundo Comhupes $^{17}$, pacientes alérgicos às cefalosporinas devem usar, como antibioticoprofilaxia, esquemas isolados ou combinados de metronidazol, clindamicina e gentamicina.

Quanto aos dias de internação dos participantes da pesquisa, foram contados e considerados aqui a variação mínima (1 dia) e variação máxima (39 dias) a partir do pósoperatório, obtendo-se a média de $2,91( \pm 3,65)$ dias. Neste estudo, foi considerado o tempo de permanência após a cirurgia até a alta medica, não caracterizando se houve ou não ISC após a alta hospitalar.

Feitosa $^{20}$ em seu estudo evidenciou que o período de internação pós-operatório para paciente com ISC variou de 9 a 123 dias, com uma média de 34 dias. Enquanto, para os pacientes sem ISC, a média de internamento pós-operatória foi de18 dias, variando de quatro a 41 dias. 
Na caracterização dos participantes segundo Infecção de sítio cirúrgico, $315(98,4 \%)$ da amostra não apresentaram infecção durante o período de internação, enquanto $5(1,6 \%)$ apresentaram ISC. Em concordância com o estudo de Gebrim ${ }^{3}$, sobre a análise da profilaxia antimicrobiana este apresentou incidência de $10 \%$ da amostra de casos de ISC, enquanto $90 \%$ não tiveram ISC. Entretanto, Oliveira ${ }^{12}$, em seu estudo notificou $25 \%$ de ISC durante a internação.

A infecção de sítio cirúrgico é a mais importante causa de complicações do pósoperatória no paciente cirúrgico. Em relação ao tempo de manifestação de ISC, verifica-se um consenso entre os vários autores de que um grande número de ISC ocorre até o sétimo dia pós-cirurgia. Então, percebe-se que a alta precoce pode ser um problema para a detecção das infecções que se manifestam nos primeiros dias de pós-operatório, especialmente para aqueles procedimentos cujo tempo de permanência no hospital após a cirurgia é muito curto ${ }^{21}$.

\section{CONCLUSÃO}

Perante os desfechos, conclui-se que apenas $1,6 \%$ da amostra apresentaram ISC, assim a utilização de antibioticoprofilaxia foi considerada adequada levando em conta o percentual de procedimentos que a utilizaram $98,4 \%$ e que a maioria se enquadrava em procedimentos potencialmente contaminados, sendo indispensável o uso de antimicrobiano. Embora o percentual de utilização de antibióticos e de paciente que não apresentaram ISC tenham sido coincidentes, não quis dizer que indivíduos que receberam antibiótico não desenvolveram ISC.

A ocorrência de uma ISC é multifatorial, dependente de fatores relacionados ao paciente, à equipe cirúrgica, ao procedimento e a contaminação do sítio cirúrgico por microorganismo durante a cirurgia, sendo este último o fator determinante. 
Deste modo o uso de antibioticoterapico não deve ser tratado de forma isolada afim de se prevenir ISC. É importante que haja um conjunto de cuidados identificáveis e controláveis que se estenda desde o pré até o pós-operatório, com vistas a evitar e/ou minimizar o surgimento de ISC, seja em cirurgias limpas ou potencialmente contaminada, com implementação de métodos de vigilância eficientes e precisos afim de se evitar prolongamento de internação e reabordagens cirúrgicas, o que acarreta custos hospitalares e possíveis danos físicos/psicológicos aos pacientes.

\section{REFERÊNCIAS}

1. Organização Mundial da Saúde. OMS. Segundo desafio global para a segurança do paciente: Cirurgias seguras salvam vidas (orientações para cirurgia segura da OMS). [Internet]. Nilo MS, Durán IA, tradutor. Rio de Janeiro: Organização Pan-Americana da Saúde; Ministério da Saúde; Agência Nacional de Vigilância Sanitária, 2009 [acessado 2021 Jun 10]. $216 p$.

Disponível

em:

http://bvsms.saude.gov.br/bvs/publicacoes/seguranca_paciente_cirurgias_seguras_salvam_vid $\underline{\text { as.pdf }}$

2. Garner BH, Anderson DJ. Surgical Site Infections: An Update. Infect Dis Clin North Am [periodical on the Internet]. 2016 Dec [cited 2021 Jun 09];30(4):909-929. doi: 10.1016/j.idc.2016.07.010. PMID: 27816143.

3. Gebrim CFL, Rodrigues JG, Queiroz MNR, Barreto RASS, Palos MAP. Análise da profilaxia antimicrobiana para a prevenção da infecção do sítio cirúrgico em um hospital do Centro-Oeste Brasileiro. Cienc Enferm [periódico na Internet]. 2014 Ago [acessado 2021 Jun 09];20(2):103-15. doi: http://dx.doi.org/10.4067/S0717-95532014000200011 
4. Kolasiński W. Surgical site infections - review of current knowledge, methods of prevention. Pol Przegl Chir. [periodical on the Internet]. 2018 Nov [cited 2021 Jun 09]6;91(4):41-47. doi: 10.5604/01.3001.0012.7253. PMID: 31481640.

5. Frasão G. Em oito meses, número de cirurgias eletivas cresceu 39,1\% no Brasil. Ministério da Saúde [Internet]. 2017 [acessado 2021 Jun 10]: [cerca de 1 tela]. Disponível em: https://antigo.saude.gov.br/noticias/agencia-saude/42101-em-oito-meses-numero-decirurgias-eletivas-cresceu-39-1-no-brasil

6. Carvalho RLR. Fatores de risco para infecção de sítio cirúrgico em procedimentos gerais em um hospital público de Belo Horizonte. [Dissertação na Internet]. Minas Gerais, Belo Horizonte: Universidade Federal de Minas Gerais, 2014 [acessado 2021 Jun 10]. 105 p. Disponível em: $\quad$ https://repositorio.ufmg.br/bitstream/1843/BUOS9KRHFA/1/disserta o_rafael_carvalho pronta.pdf

7. Longo ART, Ribeiro DR. Hipotermia como fator de risco para infecção de sítio cirúrgico: conhecimento dos profissionais de enfermagem de nível médio. Rev Min Enferm [periódico na Internet]. 2011 Jan-Mar [acessado 2021 Jun 09];15(1):34-41. Disponível em: http://www.reme.org.br/artigo/detalhes/5

8. Dhanasekaran D, Thajuddin N, Panneerselvam A. Antimicrobials: Synthetic and Natural Compounds. 1. ed. Boca Raton: CRC Press, 2015.

9. Brasil. Agência Nacional de Vigilância Sanitária. Série: Segurança do paciente e qualidade em serviços de saúde: Medidas de prevenção de infecção relacionada à assistência à saúde. Anvisa [Internet]. 2013 [acessado 2021 Jun 10].92p. Disponível em: https://www.segurancadopaciente.com.br/wp-content/uploads/2015/09/ebook-anvisa-04$\underline{\text { medidas-de-prevencao-de-de-infeccao-relacionada-a-assistencia-a-saude.pdf }}$ 
10. Kessel AS, Sharland M. The new UK antimicrobial resistance strategy and action plan. BMJ. Clinical Research ed. [periodical on the internet] 2013 Mar [cited 2021 Jun 09];11;346:f1601. doi: 10.1136/bmj.f1601

11. Brasil. Agência Nacional de Vigilância Sanitária. Série: Segurança do paciente e qualidade em serviços de saúde. Critérios Diagnósticos de Infecção Relacionada à Assistência à Saúde, Brasília: Anvisa [Internet]. 2013 [acessado 2021 Jun 10].84 p. Disponível em: http://bvsms.saude.gov.br/bvs/publicacoes/criterios_diagnosticos_infeccoes_assistencia_saud e.pdf

12. Oliveira AC de, Gama CS. Evaluation of surgical glove integrity during surgery in a Brazilian teaching hospital. Am J Infect Control [periodical on the Internet] 2014 Oct [cited 2021 Jun 09];42(10):1093-6. doi: 10.1016/j.ajic.2014.06.021

13. Santos PVF, Jesus KB, Santana KISP, Nogueira EC, Cariri LS, Brito FPG. Infecção do sítio cirúrgico em pacientes no pós-operatório de cirurgias ortopédicas eletivas. Rev Interfaces Científicas - Saúde e Ambiente [periódico na Internet]. 2017 Fev [acessado 2021 Jun 09]; 5(2):71-79. doi: https://doi.org/10.17564/2316-3798.2017v5n2p71-79

14. Brasil. Ministério da Saúde. Conselho Nacional de Saúde. Resolução 466, de 12 de dezembro de 2012. Aprova as diretrizes e normas regulamentadoras de pesquisas envolvendo seres humanos. Diário Oficial da União [Internet]. 2013 Jun 13 [acessado 2021 Jun 09]. (Seção 1):59. Disponível em: http://conselho.saude.gov.br/resolucoes/2012/Reso466.pdf 15. Gouvêa M, Novaes CO, Iglesias AC. Avaliação da antibioticoprofilaxia em pacientes cirúrgicos no Hospital Universitário Gaffrée e Guinle. Rev Col Bras Cir [periódico na Internet]. 2016 Jul-Ago [acessado 2021 Jun 09];43(4):225-234. doi: https://doi.org/10.1590/0100-69912016004001 
16. Tostes MFP, Maran E, Raimundo LS, Mai LD. Prática da profilaxia antimicrobiana cirúrgica como fator de segurança do paciente. Rev Sobecc [periódico na Internet]. 2016 Jun [acessado 2021 Jun 09];21(1):13-21. doi: https://doi.org/10.5327/Z1414-4425201600010003 17. Complexo Hospitalar Universitário Professor Edgard Santos (COMHUPES). Profilaxia Antimicrobiana Perioperatória. Ebserh Bahia [Internet]. 2013 Ago [acessado 2021 Jun 10]. Disponível em: http://www2.ebserh.gov.br/documents/1975526/2520527/diretriz_16_profilaxia_antimicrobia na_perioperatoria_revisada.pdf/bd6a4b1c-b2ca-4b9e-81f6-1cade7bc145f

18. Melo MS, Carvalho TA, Mattos MCT, Campos MPA, Mendonça SB, Lobo IMF. Avaliação da profilaxia antimicrobiana cirúrgica em um hospital de ensino. Rev Epidemiol Controle Infecç [periódico na Internet]. 2019 Jan-Mar [acessado 2021 Jun 09];9(1):75-80. doi: http://dx.doi.org/10.17058/reci.v9i1.11461

19. Silva A. Avaliação do uso de antibioticoprofilaxia em cirurgias realizadas em hospital do sul de Santa Catarina. [Dissertação na Internet]. Santa Catarina, Tubarão: Universidade do Sul de Santa Catarina. 2016 [acessado 2021 Jun 10]. Disponível em: https://www.riuni.unisul.br/bitstream/handle/12345/2037/Disserta\%C3\%A7\%C3\%A3o\%20d e\%20Ci\%C3\%AAncias\%20da\%20Sa\%C3\%BAde\%20\%20Alexandre\%20da\%20Silva.pdf? sequence=3\&isAllowed=y 20. Feitosa RGF, Fernandes FAM, Júnior ONA, Costa FA, Cavalcante LDW. Análise da incidência de infecção de sítio cirúrgico em cirurgias oncológicas do aparelho digestivo no Hospital Geral de Fortaleza. Rev Medicina Ribeirão Preto [periódico na internet]. 2014 [acessado 2021 Jun 09];47(2):157-164. doi: https://doi.org/10.11606/issn.2176$\underline{\text { 7262.v47i2p157-164 }}$ 
21. Oliveira AC, Ciosak SI. Infecção de sítio cirúrgico em hospital universitário: vigilância pós-alta e fatores de risco. Rev Esc Enferm USP [periódico na Internet]. 2007 Jun [acessado 2021 Jun 09];41(2):258-263. doi: https://doi.org/10.1590/S0080-62342007000200012 